\title{
Ultrasound-Guided 50\% Ethyl Alcohol Injection for Patients With Malleolar and Olecranon Bursitis: A Prospective Pilot Study
}

\author{
Ji Seong Hong, $\mathrm{MD}^{1}$, Hyoung Seop Kim, $\mathrm{MD}^{2}$, Jin Hyung Lee, $\mathrm{MD}^{3}$ \\ ${ }^{1}$ Department of Physical Medicine and Rehabilitation, Hando Hospital, Ansan; \\ ${ }^{2}$ Department of Physical Medicine and Rehabilitation, National Health Insurance Service Ilsan Hospital, Goyang; \\ ${ }^{3}$ Department of Rehabilitation Medicine and Research Institute of Rehabilitation Medicine, \\ Yonsei University College of Medicine, Seoul, Korea
}

Objective To evaluate the feasibility and effect of ultrasound-guided ethyl alcohol injection on malleolar and olecranon synovial proliferative bursitis.

Methods Twenty-four patients received ultrasound-guided 50\% diluted ethyl alcohol injection at the site of synovial proliferative bursitis after aspiration of the free fluid.

Results Swelling and symptoms significantly decreased in 13 of the 24 patients without any complications. Eleven patients had partial improvement in swelling and symptoms.

Conclusion Ultrasound-guided alcohol injection could be an alternative therapeutic option before surgery in patients with chronic intractable malleolar and olecranon synovial proliferative bursitis.

Keywords Synovium, Bursitis, Ethyl alcohol, Ultrasonography

\section{INTRODUCTION}

Bursae are located between surfaces where there is friction and movement. The function of the bursa is to reduce friction between two layers [1]. On ultrasound study, synovial membranes of the bursa are usually not

Received July 10, 2015; Accepted September 8, 2015

Corresponding author: Hyoung Seop Kim

Department of Physical Medicine and Rehabilitation, National Health Insurance Service Ilsan Hospital, 100 Ilsan-ro, Ilsandong-gu, Goyang 10444, Korea

Tel: +82-31-900-0114, Fax: +82-31-900-0049, E-mail: iskrakhs@gmail.com

@ This is an open-access article distributed under the terms of the Creative Commons Attribution Non-Commercial License (http://creativecommons. org/licenses/by-nc/4.0) which permits unrestricted noncommercial use, distribution, and reproduction in any medium, provided the original work is properly cited.

Copyright $\odot 2016$ by Korean Academy of Rehabilitation Medicine visible. Its thickness is typically within $1 \mathrm{~mm}$ and does not exceed $2 \mathrm{~mm}$ under normal conditions [1]. Repeated micro-trauma or inflammatory conditions induce the proliferation of synovial cells, and subsequently the synovial sac fills with fibrous exudates [2]. When the synovium proliferates to form a polyp, it is called a pannus [3]. Bursitis with synovial proliferation accompanied by vessel infiltration is hard to treat [3], and surgery is considered when conservative treatments such as steroid injections or other treatments fail [2]. Despite the use of several conservative treatments, the rate of recurrence is high $[4,5]$, and thus, many patients are not satisfied with conservative treatment.

Highly concentrated alcohol causes the denaturation of proteins [6]. Alcohol installation is used to manage 
hepatocellular carcinoma and arteriovenous malformation in medical fields $[7,8]$. Ultrasound-guided or electromyography-guided alcohol injection is commonly used in rehabilitation departments, such as a nerve block in spastic cerebral palsy patients or to improve gait patterns in hemiplegic patients or to prevent joint contracture [9].

In our study, alcohol injection was performed in 24 synovial proliferative bursitis patients before surgery was considered.

\section{MATERIALS AND METHODS}

Between October 2012 and June 2014, 24 patients were diagnosed with malleolar bursitis or olecranon bursitis based on clinical symptoms, physical examination and radiological evaluation. In all patients, steroid injection had been previously performed to treat bursitis, but all patients were consistently unresponsive to conservative treatment for at least 2 weeks. The failure of steroid injection was defined as when swelling remained the same compared to the initial state and when the patient was unsatisfied with steroid injection. Exclusion criteria included patients with diabetic ulcer, septic bursitis accompanied by local infection or skin issues, autoimmune disease such as rheumatoid arthritis or systemic lupus erythematosus, penicillin allergic patients, and those who refused alcohol injection. Our study received approval from the Institutional Review Board. All patients were informed of the medical procedure and their informed consent obtained. Patient medical records, age, gender, the side of affected bursa, disease duration, occupation type, comorbidity, clinical outcome, trauma history, number of alcohol treatments and complications after injections were collected. We carried out the investigations and sonoguided procedure with the GE Voluson i ultrasound machine (GE Healthcare Technologies, Milwaukee, WI, USA). A 5-13 MHz linear array 2D transducer was applied. All physical examinations as well as ultrasonography and ultrasonoguided 50\% ethyl alcohol injections were performed by a single physician. Synovial proliferative bursitis was diagnosed when there was cystic fluid collection or lobulated synovial thickening surrounded by hypoechoic fluid and when there was active inflammation on an active Doppler signal. After the location of a bursa sac was identified through ultrasound, all the patients underwent ultrasound-guided aspiration with an 18-gauge needle. Then, $2 \%$ lidocaine was aspirated to the bursa sac for local anesthesia. After the aspiration of $2 \%$ lidocaine, a mixture of $50 \%$ dehydrated ethyl alcohol $2.5 \mathrm{~mL}$ and normal saline $2.5 \mathrm{~mL}$ was injected into the bursa sac. Next, the process of injecting normal saline and aspirating was repeated similar to irrigation. After performing the process for a minute, we confirmed that as much ethyl alcohol as possible had been removed from the bursa sac. After the injection, compressive dressing was undertaken using an elastic bandage. During follow-up at 1-week intervals, the procedure was repeated if the fluid collection was not reduced or if there was no effect. To check for recurrence or side effects after injection, we followed the patients in 3 months intervals until at least 1 year.

The clinical outcomes were assessed using the response evaluation criteria, which were modified from the guidelines of the response to treatment in a solid tumor [10]. Each assessment was performed by one of the authors who had not performed the injection. The response evaluation criteria included the degree of fluctuation, shrinkage of the bursal sac and soft tissue swelling (Table 1). In other words, the complete response was defined as when swelling was not found in the bursa sac, when no fluid collection was visible on the ultrasound and when the patient was satisfied with his or her status (Fig. 1). A partial response was defined as when bursa swelling was not visually clear, when minimal fluid collection was visible on the ultrasound, needle aspiration was not possible and when the patient was satisfied with the treatment. No response was defined as when the patient was unsatisfied with the treatment and not much change was found in the fluid collection on the ultrasound. Side effects, such as pain, fever, and scarring were also evaluated during the follow-up periods. To manage possible side effects,

Table 1. Response evaluation criteria for malleolar or olecranon bursitis

\begin{tabular}{clcl}
\hline Response & Shrinkage & Fluctuation & $\begin{array}{c}\text { Soft tissue } \\
\text { swelling }\end{array}$ \\
\hline CR & Complete & No & None or minimal \\
PR & Partial & Yes & Yes \\
NR & No change & Yes & Yes \\
\hline
\end{tabular}

CR, complete response; PR, partial response; NR, no response. 


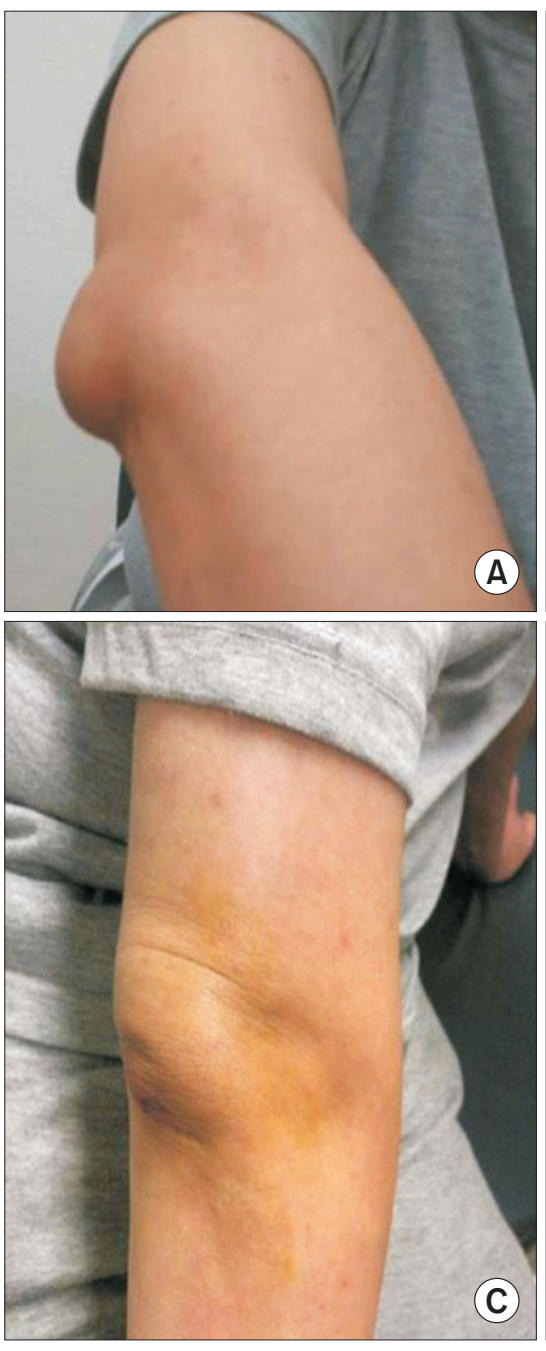

(A)
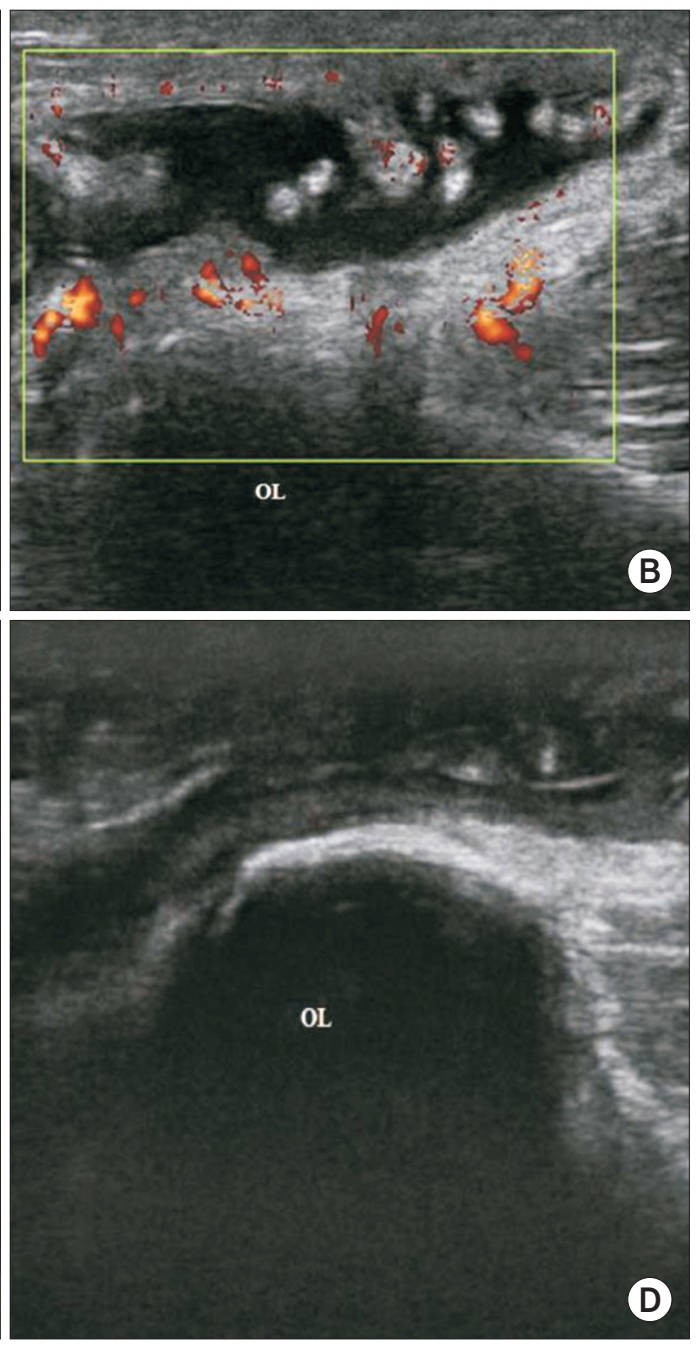

Fig. 1. Ultrasound image and photographs of the olecranon (OL) bursitis. (A) Photograph of fluid collection prior to injection. (B) Initial ultrasound image of olecranon bursa. Pannus proliferation inside the olecranon bursa is seen. (C) Photograph of improved fluid collection after injection. (D) Ultrasound image of the shrunk bursa. Complete response was found after injection; pannus was removed in the oleclenon bursa; no fluid collection was found. such as local heat and pain, we prescribed non-steroidal anti-inflammatory drugs such as meloxicam to all patients for 7 days after injection. After alcohol injection, follow-ups were performed at outpatient clinic. When this was impossible, recurrence or side effects were surveyed over the phone.

\section{RESULTS}

Twenty-four patients were enrolled in this study, and their average age was 62.5 years. There were 2 patients with olecranon synovial proliferative bursitis and 22 patients with malleolar synovial proliferative bursitis. Complete resolution was observed in 13 patients (54\%) who showed a response after the first alcohol injection; 11 patients $(46 \%)$ responded after receiving the therapy two or more times (Table 2). There were no cases with
Table 2. Clinical outcomes of alcohol injection for bursitis

\begin{tabular}{|cc|}
\hline Response to treatment & No. of patients (\%) \\
\hline CR & $13(54)$ \\
PR & $11(46)$ \\
NR & 0 \\
Total & $24(100)$ \\
\hline
\end{tabular}

$\mathrm{CR}$, complete response; PR, partial response; NR, no response.

no response. The mean follow-up period was $16 \pm 5.01$ months. As the injection trial number varied in our patients the follow-up period also differed. Ten patients had comorbidity such as hypertension, diabetes mellitus and hyperlipidemia (Table 3 ). However, the clinical outcomes might not be related to comorbidity. Four patients (16.6\%) showed minor side effects and their average 


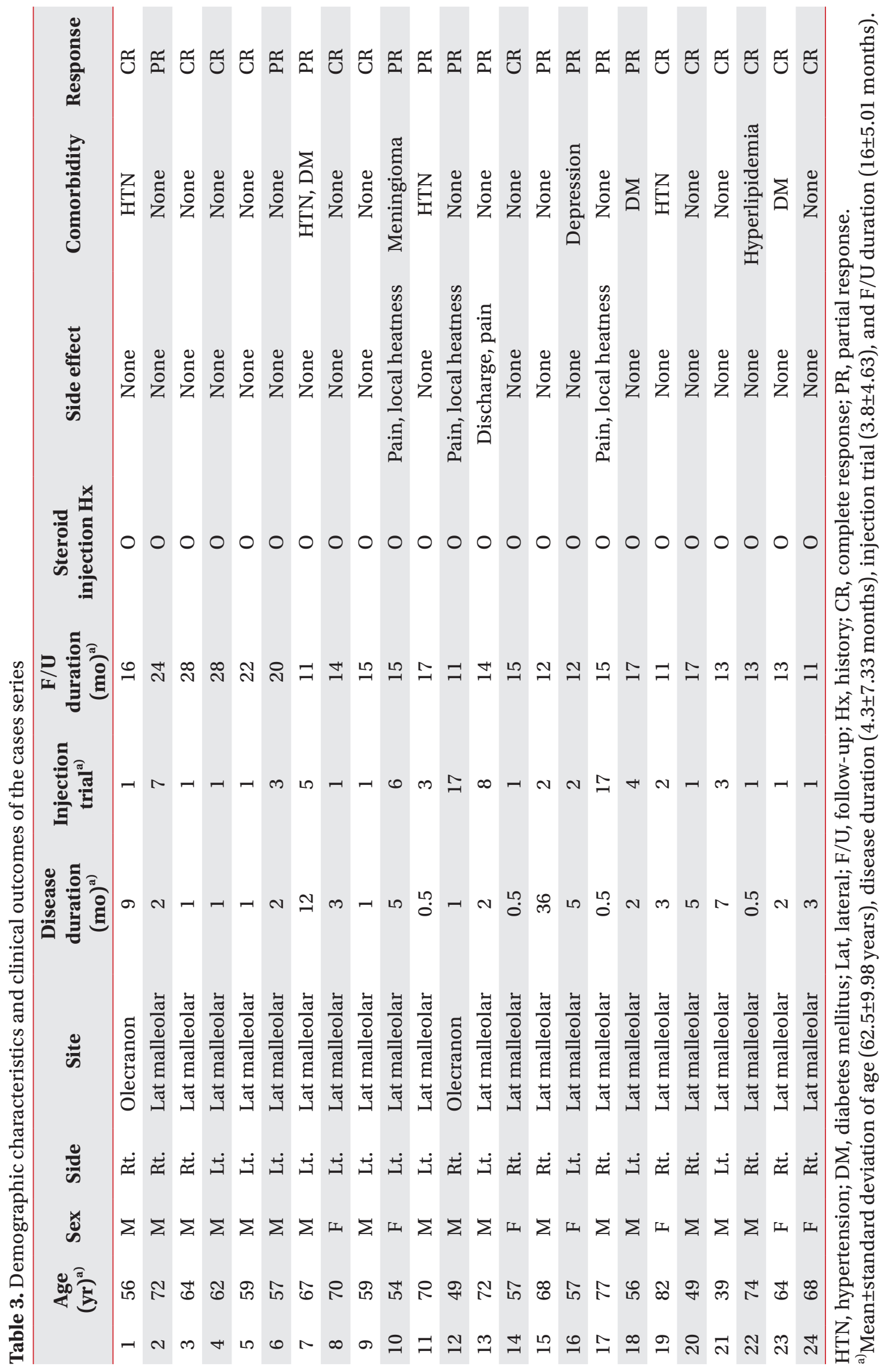


symptom period was $2.1 \pm 2.0$ months. However, they were all well-controlled after the administration of antipyretic medications (Table 4). There were 14 patients (58\%) whose symptom duration was less than 3 months. The mean alcohol injection trial for symptom periods lasting more than 3 months (chronic period) was $2.4 \pm 1.7$, while the mean trial for symptom periods lasting less than 3 months (acute period) was $4.7 \pm 5.5$ (Table 5).

Table 4. Side effects of alcohol injection for bursitis

\begin{tabular}{lc}
\hline \multicolumn{1}{c}{ Side effect } & No. of patients (\%) \\
\hline Local heat with tolerable pain & $4(16.6)$ \\
Numbness & 0 \\
Skin pigmentation & 0 \\
Scar or deformity & 0 \\
\hline
\end{tabular}

\section{DISCUSSION}

Bursae are located between surfaces where there is friction and movement, often between different tissues (e.g., tendon and bone). Typically, 1-2 mm thick bursae exist in the body where protective responses are needed for repeated traumas or frictions $[1,11]$. On a normal ultrasound study, synovial membranes are not observed.

Table 5. Mean number of trials for alcohol injection compared by symptom duration

\begin{tabular}{lc}
\hline \multicolumn{1}{c}{ Symptom duration } & $\begin{array}{c}\text { Mean no. of trials } \\
\text { for alcohol injection }\end{array}$ \\
\hline$<3$ mo (acute period) & $4.7 \pm 5.5$ \\
$\geq 3$ mo (chronic period) & $2.4 \pm 1.7$ \\
\hline
\end{tabular}
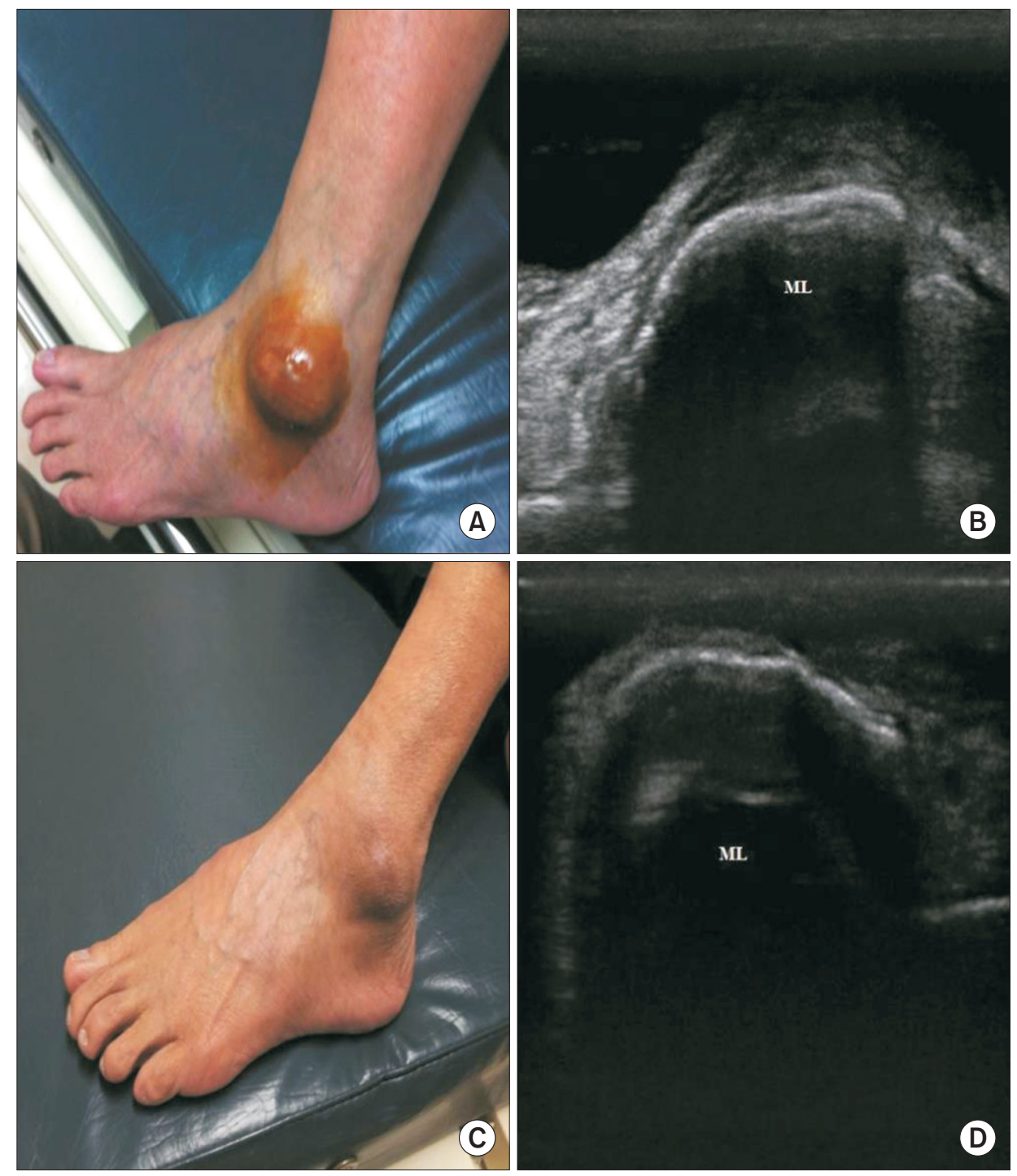

Fig. 2. Ultrasound image and photographs of the lateral malleolar bursitis. (A) Photograph of fluid collection prior to injection. (B) Initial ultrasound image of lateral malleolar bursa showing fluid collection. (C) Photograph of improved fluid collection after injection. (D) Ultrasound image of the bursa after injection showing improved status of fluid collection. ML, malleolar bone. 
Bursitis undergoes synovial proliferation under repeated micro traumas or inflammatory conditions while the synovial sac is packed full of fibrous exudates [2]. In this study, the improvement of fluid collection in the ultrasounds of patients with malleolar bursitis or olecranon bursitis was studied. The bursitis were vulnerable to repeated micro traumas rather than inflammatory conditions such as autoimmune diseases (Figs. 1, 2).

Non-surgical treatments for bursitis include conservative approaches including pain relief or ice pack application. However, they can only reduce inflammation and have difficulty lowering bursa fluid collection $[11,12]$. Steroid injection is possible, but synovial proliferative bursitis with steroid injection is accompanied by a risk of fat atrophy and depigmentation of skin tissues, infection, tendon rupture, hyperglycemia and iatrogenic Cushing syndrome. In addition, there is a high likelihood of relapse when re-injection is difficult due to the above side effects $[4,5,13]$. Additionally, this synovial proliferation accompanied by vessel proliferation is hard to treat, and surgery is used when conservative treatment is not useful. In the event of surgical treatment, incision and drainage are performed if the former does not result in any progress [14]. However, this method reportedly involves operative complications such as painful scarring, superficial peroneal nerve injury, cosmetic problems, fistula and local infection [15-18]. Furthermore, Lee et al. [19] reported that the non-surgical leakage of exudates in patients with chronic lateral malleolar bursitis using an indwelling silk suture resulted in complications such as skin redness and recurrence. In addition, Park et al. [20] reported on the treatment of malleolar bursitis in 20 patients after injecting OK-432, an immunotherapeutic agent commonly used for head and neck cancer. However, OK-432 was found to have side effects such as the compression of surrounding tissues due to severe soft tissue swelling [21].

The medical application of alcohol injection is mainly found in treatments for arteriovenous malformation and cancer such as hepatoma [7,8]. In rehabilitation medicine, it is used for nerve block in spastic cerebral palsy patients, to improve gait patterns in hemiplegic patients or to prevent joint contracture [9]. Some complications of alcohol injection have been reported such as skin color changes, infection, nerve injury, ulcer and abscess formation, but none had any severe complications.
We have experience with treating subdeltoid bursitis and finger isolated tenosynovitis with $50 \%$ ethyl alcohol $[22,23]$. Taking into consideration the results of our previous studies and the 24 cases in this study, alcohol injection could be a treatment option for synovial proliferative bursitis without surgery. Alcohol injection has fewer complications and no surgical scarring [8]. In our patients, there were no specific complications probably because alcohol dilution was also performed, decreasing the possibility of focal or general complications. Alcohol injection for synovial proliferative bursitis had a good prognosis in 13 out of 24 cases (Table 2). In addition, alcohol injection at outpatient clinics is relatively cost effective compared to other operative treatments. According to Part et al. [20], full recovery of malleolar bursitis following a single injection of OK-432 was observed in 16 cases, and full recovery after two injections was observed in 4 cases. However, our cases involved a large number of treatments, up to as many as 17 injections. This could be attributed to the $50 \%$ alcohol concentration. In general, the ethyl alcohol concentration used in the treatment of vascular malformation and hepatocellular carcinoma is 95\%. When 95\% alcohol is injected, the vascular endothelium is destroyed and red blood cell hemolysis occurs, which induces blood coagulation [8].

Therefore, $50 \%$ alcohol is believed to be a low enough concentration to destroy abnormal vasculature and proliferative pannus extending to the surrounding area of the bursa and therefore, requires several injections. In future studies, a range of concentrations for ethyl alcohol needs to be tested to determine the effective concentration and efficacy.

The mean number of injection trials was $3.8 \pm 4.63$ in our study. As the injection trial number varied, the follow-up period ranged from a minimum of 11 months to a maximum of 28 months. Alcohol injection can be continuously performed if there is no side effect. According to our results, alcohol injection should be done at least 4 times to obtain therapeutic effects.

The mean alcohol injection trial number during the chronic period was lower than during the acute period (Table 5). According to this result, as fewer injection trials are needed, alcohol injection is more effective in chronic period patients compared to acute period patients. The average symptom period for patients who had side effects was $2.1 \pm 2.0$ months. Therefore, side effects also had a 
greater tendency to appear in the acute period patients. Alcohol injection should be performed for chronic intractable patients who show no response to conservative treatments including steroid injection. The strength of the alcohol injection in our study was the low recurrence rate. All patients showed no recurrence within a followup period of $16 \pm 5.0$ months. A large number of studies can offer guidelines and protocols for alcohol injection therapy.

Among the 24 patients in this study, 3 had been on medication such as aspirin or Plavix for bleeding. However, all of them showed no signs of bleeding under ultrasound guided aspiration (Table 3). When the first injection did not result in complete response and a single injection did not lead to full recovery, bloody exudates were found during the second aspiration, regardless of the use of aspirin or Plavix. In addition, unlike Westerners, Koreans usually sit crossed-legged on the floor and are more likely to hit the malleolar bursa. This may hinder immediate recovery after the operation.

The findings of this study suggest that compared to anticancer drugs, $50 \%$ ethyl alcohol injection has advantages such as lower cost and fewer side effects. We can also assume that compared to conventional treatment such as steroid injection, the denaturation effect of alcohol is more effective in destroying synovial sac cells. Biopsy of the synovial cell was not conducted in this study but comparing the biopsy results with conventional treatment groups would be useful in a future study.

The disadvantages include having to perform multiple injections for complete recovery rather than just one or two injections.

The limitation of this study lies in the inclusion of acute period patients. As our study shows that alcohol injection has a lower effect and more side effects in the acute period, acute period patients should had been excluded. Acute period patients should attempt conservative treatment for at least 3 months before trying alcohol injection. There is a need to perform a large number of alcohol injections in a random control case study to compare the effects of alcohol injection, conventional treatment and surgical treatment in order to set a guideline for malleolar and olecranon synovial proliferative bursitis treatment. Second, the malleolar bursa and olecranon bursa that we examined did not communicate with the joint but were close to the skin. Therefore, those that do communicate with joints, such as the trochanteric bursa, suprapatellar bursa, and iliopsoas bursa or bursitis, with a relatively deep portion that were not studied are not applicable. Third, the number of steroid injection trials and the recurrence after steroid injection was not evaluated. As there was no control group for comparison with the steroid injection group, a case control study including steroid injection will be needed in the future.

Conventional treatments for malleolar or olecranon proliferative bursitis include medication, steroid injection, sclerotherapy and surgery. However, these methods all involve recurrence or complications. This study reports improvements in the symptoms of patients diagnosed with malleolar or olecranon proliferative bursitis following alcohol injections and was visualized by ultrasound. There is a need to investigate the injection of high-concentration ethyl alcohol in the future. In conclusion, ultrasound guided $50 \%$ alcohol injection could be an alternative therapeutic option before surgery in patients with chronic intractable malleolar and olecranon synovial proliferative bursitis.

\section{CONFLICT OF INTEREST}

No potential conflict of interest relevant to this article was reported.

\section{REFERENCES}

1. Bureau NJ, Dussault RG, Keats TE. Imaging of bursae around the shoulder joint. Skeletal Radiol 1996;25:513-7.

2. Baker CL Jr, Massie RV, Hurt WG, Savory CG. Arthroscopic bursectomy for recalcitrant trochanteric bursitis. Arthroscopy 2007;23:827-32.

3. Van Holsbeeck MT, Introcaso JH. Musculoskeletal ultrasound. St. Louis: Mosby; 2001.

4. Avci S, Sayli U. Lateral premalleolar bursitis as a result of sitting on the foot. Foot Ankle Int 2001;22:64-6.

5. Choi JH, Lee KT, Lee YK, Kim DH, Kim JR, Chung WC, et al. Endoscopic versus open bursectomy of lateral malleolar bursitis. Knee Surg Sports Traumatol Arthrosc 2012;20:1205-8.

6. Lobert S. Ethanol, isopropanol, methanol, and ethylene glycol poisoning. Crit Care Nurse 2000;20:41-7.

7. Berenguer B, Burrows PE, Zurakowski D, Mulliken JB. Sclerotherapy of craniofacial venous malforma- 
tions: complications and results. Plast Reconstr Surg 1999;104:1-15.

8. Lee CH, Chen SG. Direct percutaneous ethanol instillation for treatment of venous malformation in the face and neck. Br J Plast Surg 2005;58:1073-8.

9. Chua KS, Kong KH. Alcohol neurolysis of the sciatic nerve in the treatment of hemiplegic knee flexor spasticity: clinical outcomes. Arch Phys Med Rehabil 2000;81:14325.

10. Therasse P, Arbuck SG, Eisenhauer EA, Wanders J, Kaplan RS, Rubinstein L, et al. New guidelines to evaluate the response to treatment in solid tumors. J Natl Cancer Inst 2000;92:205-16.

11. Brown TD, Varney TE, Micheli LJ. Malleolar bursitis in figure skaters: indications for operative and nonoperative treatment. Am J Sports Med 2000;28:109-11.

12. Smith A. Skating injuries: a guide to prevention and management. J Musculoskelet Med 1997;14:10-30.

13. Butcher JD, Salzman KL, Lillegard WA. Lower extremity bursitis. Am Fam Physician 1996;53:2317-24.

14. Stewart NJ, Manzanares JB, Morrey BF. Surgical treatment of aseptic olecranon bursitis. J Shoulder Elbow Surg 1997;6:49-54.

15. Kim MG, Kim SG, Lee JH, Eun YG, Yeo SG. The therapeutic effect of OK-432 (picibanil) sclerotherapy for benign neck cysts. Laryngoscope 2008;118:2177-81.

16. Kubota T, Ohta N, Fukase S, Kon Y, Aoyagi M. Treatment of auricular hematoma by OK-432. Otolaryngol Head Neck Surg 2010;142:863-6.

17. Ogose A, Hotta T, Kawashima H, Endo N. A painful large ganglion cyst of the ankle treated by the injection of OK432. Mod Rheumatol 2007;17:341-3.

18. Ohta N, Fukase S, Watanabe T, Ito T, Aoyagi M. Effects and mechanism of OK-432 therapy in various neck cystic lesions. Acta Otolaryngol 2010;130:1287-92.

19. Lee BJ, Lee SR, Kim ST. The effect of indwelling silk suture following aspiration in the treatment of chronic lateral malleolar bursitis. J Korean Foot Ankle Soc 2005;9:38-41.

20. Park KH, Lee J, Choi WJ, Lee JW. OK-432 sclerotherapy for malleolar bursitis of the ankle. Foot Ankle Int 2013;34:1389-94.

21. Laranne J, Keski-Nisula L, Rautio R, Rautiainen M, Airaksinen M. OK-432 (Picibanil) therapy for lymphangiomas in children. Eur Arch Otorhinolaryngol 2002;259:274-8.

22. Shin JB, Joo SH, Kim HS, Cho HK. Treatment of synovial proliferative subdeltoid bursitis through alcohol installation: a case report. J Korean Acad Rehabil Med 2010;34:106-9.

23. Shin JE, Park JH, Yi HS, Ye BK, Kim HS. Treatment of chronic isolated finger flexor tenosynovitis through $50 \%$ dehydrated alcohol installation. Ann Rehabil Med 2013;37:586-90. 